\title{
Advances of Peach Breeding in China
}

\author{
Wang Zu-Hua and Lu Zhen-Xiang \\ Institute of Horticulture, Jiangsu Academy of Agricultural Sciences, Nanjing, Jiangsu 210014 \\ Peoples Republic of China
}

China is the birthplace of peach [Prunus persica (L.) Batsch.]. On the basis of the productive status of peach, its geographical distribution, biological characteristics, and ecological relations, China can be divided into five regions suitable for peach cultivation: the northwest drought plateau; the north China plain; the Changjiang River valley; the Yunnan-Kuizhou plateau and the Qinghai-Xizang frigid plateau; and two suitable subregions: the northeast frigid plateau and the south China subtropical region (Wang Zuhua et al., 1990a). Through the ages, rich and special cultivars (or strains) have been developed as the result of domestication and cultivation (Li Zai-Long, 1984). According to their fruit characteristics, peach cultivars can be classified into six groups: crisp (YingRou-Tao); honey (Mi-Tao); juicy (Shui-MiTao); flat (Pan-Tao); the nectarine (You-Tao) and yellow peach (Huang-Tao) (Wang Zuhua et al., 1990b).

Peach improvement had been carried out unconsciously long before 1949 in China (Shen Dexu, 1980). In the 1950s, general investigations of peach germplasm were conducted throughout the country, and excellent cultivars became available for local peach production ( $\mathrm{Yu}$ Chengzhe and Zong Xuepu, 1980). In the northwest, north, and east of China, several foreign cultivars (mostly from Japan) were introduced and used successfully (Wang Zuhua et al., 1989). Meanwhile, Chinese peach breeders engaged mainly in selecting better strains from hybrid seedlings of peach cultivars with open pollination (Shen Dexu et al., 1962). It was not until the early 1960 s that peach-breeding programs in China were systematically initiated (Shen Dexu, 1980; Wang Zuhua and Duan Xinmei, 1964). Northwestern Agriculture Univ. (Xian), Beijing Agricultural Univ. (Beijing), and Jiangsu Academy of Agricultural Sciences (Nanjing) were the agencies that carried out early programs, followed by Zhejiang Agricultural Univ. (Hangzhou), Zhejiang Academy of Agricultural Sciences (Hangzhou), Beijing Academy of Agricultural and Forestry Sciences (Beijing), Zhengzhou Fruit Research Institute (Zhengzhou), Dalian Agricultural Research Institute (Dalian), and Shanghai Academy of Agricultural Sciences (Shanghai) (Wang Zuhua

Received for publication 12 June 1991. Accepted for publication 9 Oct. 1991. This manuscript is dedicated to the late Wang $\mathrm{Zu}-\mathrm{Hua}$ for his extraordinary contributions to peach breeding. I thank D.W. Rammng and Jin Zewan for their assistance in revising this manuscript. et al., 1989). There are now more than 10 agricultural academies (or universities) engaged in peach breeding. Six national peach breeding symposia have been held since the 1960 s to promote national exchange and cooperation. The painstaking efforts of breeders have resulted in remarkable advances in peach breeding. The release and use of many new cultivars (or strains) for dessert use and the canning industries has greatly increased peach production and quality in China (Wang Zuhua et al., 1989).

\section{Breeding for dessert peaches}

In the beginning, Chinese peach breeders paid considerable attention to the selection of early maturing cultivars. There were only a few early maturing cultivars available for production in the 1960s; mainly crisp peaches, such as 'Huo Zhu', 'Su Hong', 'Wu Yue Xian', as well as some juicy peaches introduced from abroad, such as 'Amsden June', 'Wasesuimitsu', 'Kobayashi', 'Hakkobi'; however, their fruit quality was low. Therefore, the late-maturing traditional cultivars (such as 'Bai Hua', 'Wu Yun') and the midor early maturing cultivars (such as 'Okubo', 'Amsden June') were used as crossbreeding parents, and many hybrid seedlings were evaluated. By the 1970 s, many new early maturing cultivars, such as 'Mai Xiang', 'Zao Xiang Yu', 'Qing Feng', 'Bei Nong Zao Yan', 'Bei Nong 2', 'Jin Yan', 'Xi Nong Zao Mi', 'Hang Zhou Zao Shui Mi', 'Xue Yu Lu', 'Zhong Shan Zao Lu', 'Yu Hua Lu', 'Zhao Xia' etc., had been released $(\mathrm{Hu}$ Niyun and Lu Guangming, 1988; Hu Zhengling et al., 1989; Wang Zuhua et al., 1990a) (Table 1). Those ripening within 80 days from anthesis, with an average fruit weight $>100$ $\mathrm{g}$, and a soluble solids concentration (SSC) $>9 \%$ to $10 \%$, had almost completely replaced the former cultivars and played an important role in dessert peach production by the 1980 s.

On the basis of the above achievement, attempts to develop extra-early maturing peaches was made (Zhuang Enji et al., 1982; Wang Zuhua et al., 1989). To germinate hybrid seeds from early maturing cultivars, breeders have researched embryo culture since the late 1970s (Hu Zhengling et al., 1981; Yang Zenhai et al., 1983; Zhu Jijin et al., 1983). Young embryos [ <0.30 PFl (embryo length/seed length)] from extra-early maturing cultivars have been successfully cultured with the improved culture media and environment, by culturing embryos from fruit at the firm-ripe stage (Zhu Jijin et al., 1985). The culture of immature embryos permitted the use of early maturing cultivars as seed or pollen parents. As a result, several extraearly maturing cultivars, such as 'Zao Xia Lu', 'Chun Lei', 'Chun Hua', 'Zao Hua Lu', 'Yang Zhou Zao Tian Tao', 'Rui Yu', 'Xin Xing', 'Jin Chun', 'Shuang Feng', 'Tuan Chen Zao Sheng', were released in the 1980s (Li Zengren et al., 1990; Wang Zuhua, 1988; Zhuang Enji et al., 1987; Zuo Xiangquan and Wu Yongli, 1989) (Table 1). These new cultivars, ripening at $\approx 60$ days from anthesis, with an average fruit weight $>70 \mathrm{~g}$, and SSC $>8 \%$, are now being used commercially. The in-ovulo embryo culture of extra-early maturing cultivars has been studied recently (Yao Qiang et al., 1988; Zhuang Enji et al., 1990).

Before the 1970s, the leading cultivars of midmaturing peaches in production were 'Hakuho' and 'Okubo', both introduced from Japan. 'Hakuho' was distributed widely in eastern and northern China, but its fruit was small; 'Okubo' was suitable for northern China, but tree vigor was weak. The main goals of midmaturing peach breeding programs are to develop cultivars with superior fruit quality, an average fruit weight $>120$ $\mathrm{g}$, and SSC $>10 \%$ to $12 \%$. New cultivars with these characteristics were released during the last two decades, such as ' $\mathrm{Xi}$ Nong Shui Mi', 'Ju Hong Shui Mi', 'Jing Yu', 'Yun Shu 1', 'Hang Yan', 'Yang Tao 97', 'Zhao Hui' (Chen Qihui and Gao Min, 1981; Wang Zuhua et al., 1990a) (Table 1).

Although there are many excellent latematuring conventional peach cultivars in China, such as 'Bai Hua', 'Yu Lu', 'Fei Cheng Tao', 'Shen Zhou Mi Tao', further exta-late-maturing cultivars were required to prolong the period of fresh peach supply. Furthermore, there still existed some gaps in the ripening sequence from early to late. The main objectives of the late-maturing peach breeding programs included superior fruit quality, average fruit weight $>130 \mathrm{~g}$, SSC $>12 \%$ to $13 \%$, with little premature drop before harvest. By the 1980s, several latematuring or extra-late-maturing cultivars, such as 'Jing Yan', 'Qui Xiang', 'Ba Yue Cui', 'Wan Bai Tao', 'Hang Yu', 'Wan Shuo Mi', 'Xin Bai Hua', 'Ying Qing', had been released (Wang Zuhua et al., 1990a) (Table 1).

\section{Breeding for canning peaches}

While carrying on the breeding programs for dessert peaches, Chinese peach breeders also engaged in improving canning peaches. In the 1960s, the development of yellow, nonmelting, and clingstone peaches began. The breeding goals for canning peaches were 
Table 1. Characteristics of main dessert peach cultivars in China.

\begin{tabular}{|c|c|c|c|c|c|c|}
\hline Cultivar & Parentage $^{\mathbf{z}}$ & $\begin{array}{c}\text { Maturity } \\
\text { (days) }\end{array}$ & $\begin{array}{l}\text { Mean } \\
\text { fruit wt } \\
\text { (g) }\end{array}$ & Flesh $^{\mathbf{x}}$ & $\begin{array}{c}\text { Soluble } \\
\text { solids } \\
\text { concn (\%) }\end{array}$ & Released byw \\
\hline Zao Xia Lu & Sunagowase $\times$ Yu Hua Lu & $\mathrm{EE}(54)$ & 90 & W.SM.C. & 9.0 & ZAAS \\
\hline Chun Lei & Sunaguwase $\times$ Bai Xiang Lu & $\mathrm{EE}(56)$ & 70 & W.SM.SF. & 9.5 & SAAS \\
\hline Chun Hua & Ben Nong $2 \times$ Chun Lei & $\mathrm{EE}(60)$ & 86 & W.SM.C. & 10.0 & SAAS \\
\hline Zao Hua Lu & Yu Hua Lu op. & $\mathrm{EE}(57)$ & 86 & W.SM.C. & 12.1 & JAAS \\
\hline \multicolumn{7}{|l|}{ Yang Zhou Zao } \\
\hline Tian Tao & Wu Yun $x$ Yang Tao 2 & $\mathrm{EE}(60)$ & 120 & W.HM.C. & 14.2 & YARS \\
\hline Rui Yu & Chu Xiao Mei X Amsden June & $\mathrm{EE}(57)$ & 80 & W.SM.C. & 10.1 & SxAAS \\
\hline Xin Xing & Hakuho op. & $\mathrm{EE}(62)$ & 119 & W.SM.C. & 10.5 & HAU \\
\hline Jin Chun & Zao Huang Jin op. & $\mathrm{EE}(63)$ & 131 & W.HM.C. & 9.5 & BAAFS \\
\hline Shuang Feng & Zao Xiang Yu x Okubo & $\mathrm{EE}(62)$ & 107 & W.SM.C. & 10.0 & BAAFS \\
\hline \multicolumn{7}{|l|}{ Tuan Cheng } \\
\hline Zao Sheng & Qing Feng sm. & $\mathrm{EE}(65)$ & 120 & W.SM.C. & 9.0 & HARI \\
\hline Mai Xiang & Okubo $x$ Amsden June & $E(73)$ & 102 & W.SM.C. & 9.7 & BAAFS \\
\hline Zao Xiang Yu & Okubo $\times$ Kakkobi & $E(76)$ & 100 & W.SM.C. & 12.0 & BAAFS \\
\hline Qing Feng & Okubo $\times$ Amsden June & $\mathrm{E}(78)$ & 130 & W.SM.SF. & 11.4 & BAAFS \\
\hline Bei Nong Zao Yan & Unknown & $E(74)$ & 125 & W.HM.C. & 11.0 & BAU \\
\hline Bei Nong 2 & Hakuto op. & $\mathrm{E}(80)$ & 120 & W.HM.F. & 12.2 & BAU \\
\hline Jin Yan & Okubo x Zao Xiang Yu & $E(76)$ & 128 & W.SM.C. & 12.3 & TAAS \\
\hline $\begin{array}{l}\text { Xi Nong Zao Mi } \\
\text { Hang Zhou Zao }\end{array}$ & Xi Nong Shui Mi x Amsden June & $\mathrm{E}(70)$ & 85 & W.SH.C. & 11.0 & NAU \\
\hline Shui $\mathrm{Mi}$ & Wu Yun $x$ Amsden June & $E(72)$ & 120 & W.SM.C. & 9.0 & ZAAS etc. \\
\hline Xue Yu Lu & Bei Hua x Yu Hua Lu & $\mathrm{E}(79)$ & 109 & W.SM.C. & 12.5 & ZAAS \\
\hline Zhong Shan Zao Lu & Bei Hua $\times$ Hakkobi & $E(70)$ & 102 & W.SM.SF. & 11.0 & JAAS \\
\hline Yu Hua Lu & Bei Hua $x$ Chinese Clingstone & $E(75)$ & 125 & W.SM.SF. & 11.4 & JAAS \\
\hline Zhao Xia & Bei Hua x Hakkobi & $\mathrm{E}(80)$ & 130 & W.HM.C. & 10.0 & JAAS \\
\hline Xi Nong Shui Mi & Unknown op. & $\mathrm{M}$ & 160 & W.HM.C. & 12.2 & NAU \\
\hline Ju Hong Shui Mi & Okubo sm. & M & 200 & W.HM.F. & 11.8 & Unknown \\
\hline Jin $\mathrm{Yu}$ & Okubo $\times$ Okitsu & $\mathbf{M}$ & 150 & W.HM.F. & 11.0 & BAAFS \\
\hline Yun Shu 1 & Wu Yun $x$ Xiao Shu & $\mathbf{M}$ & 100 & W.HM.C. & 11.0 & ZAAS etc. \\
\hline Hang Yan & Wu Yun $x$ Xiao Shu & M & 113 & W.HM.F. & 10.5 & ZAAS etc. \\
\hline Yang Tao 97 & Wu Yun $x$ Hakuho & M & 136 & W.HM.F. & 13.0 & YARI \\
\hline Zhao Hui & Bai Hua $\times$ Tasubanawase & M & 155 & W.HM.C. & 12.0 & JAAS \\
\hline Jin Yan & Lu Hua 5 x Okubo & $\mathbf{L}$ & 150 & W.HM.C, & 13.5 & BAAFS \\
\hline Qui Xiang & Unknown & $\mathbf{L}$ & 200 & W.HM.C. & 13.6 & Unknown \\
\hline Ba Yue Cui & Lu Hua $5 \times$ Okubo & $\bar{L}$ & 150 & W.HM.C. & 13.2 & BAAFS \\
\hline Wan Bai Tao & Chinese Clingstone sm. & $\mathrm{L}$ & 159 & W.HM.C. & 13.5 & CARI \\
\hline Hang Yu & Yu Lu op. & $\mathrm{L}$ & 125 & W.SM.C. & 14.0 & ZAAS etc. \\
\hline Wan Shuo Mi & Wan Shui Mi $\times$ Fei Cheng Tao & $\mathrm{L}$ & 222 & W.HM.C. & 15.5 & JAAS \\
\hline Xin Bai Hua & Bai Hua op. & $\overline{\mathrm{L}}$ & 192 & W.HM.C. & 17.0 & JAAS \\
\hline Ying Qing & Unknown sm. & EL & 164 & W.HM.C. & 13.0 & Unknown \\
\hline
\end{tabular}

${ }^{z}$ op., open pollination, sm., shoot mutation.

${ }^{y} \mathrm{EE}$ (extra early), $\leq 65$ days of fruit growth post-anthesis; E (early), 66-90 days; $M$ (mid), 91-115 days; L (late), 116-140 days; EL (extra late), $>140$ days.

xW., white; Y., yellow (colorimetric card grade in parentheses); SM., soft melting; HM., hard melting; NM., nonmelting; F., freestone; C., Clingstone; SF., semi-free stone.

wBAAFS = Beijing Academy of Agricultural and Forestry Sciences (Beijing, 100093). BAU = Beijing Agricultural University (Beijing, 100094); CARI = Chengdu Agricultural Research Institute (Chengdu, 61000); DARI = Dalian Agricultural Research Institute (Dalian, 116036); HAU = Hebei Agricultural University (Baoding, 071001); HARI = Haidian Agricultural Research Institute (Beijing, 100080); JAAS = Jiangsu Academy of Agricultural Scicnces (Nanjing, 210014); NAU = Northwcstcrn Agricultural University (Xian, 712100); SAAS = Shanghai Academy of Agricultural Sciences (Shanghai, 201106); SxAAS = Shanxi Academy of Agricultural Sciences (Taiyuan, 030800); TAAS = Tianjin Academy of Agricultural Sciences (Tianjin, 300112); YARI = Yangzhou Agricultural Research Institute (Yangzhou, 225002); ZAAS = Zhejiang Academy of Agricultural Sciences (Hangzhou, 310021); ZFRI = Zhengzhou Fruit Research Institute, Chinese Academy of Agricultural Sciences (Zhengzhou, 450004).

further refined in the 1970 s to select for round and uniform shape, fruit diameter $>5.5 \mathrm{~cm}$, golden-yellow to orange-yellow flesh (colorimetric card above grade 6), no red pigment in the flesh or around the stone, suitable sugar : acid ratio, agreeable flavor, and pleasant aroma (Wang Zuhua et al., 1989).

From the 1960s to mid-1970s, advances in peach breeding for canning were unsatisfactory, since few specialized cultivars for canning were available and there was little suitable germplasm that could be used as parental material (Wang Zuhua et al., 1989). Breeders believed that Europe's traditional yellow cultivars, such as 'Phillips' and 'Tuscan', have fruit characteristics suitable for use in breeding programs, but they were not very adaptable and productive in China, especially in eastern China. Others, such as 'Elberta' and 'Early Crawford', were more adaptable and productive, but they are melting and freestone. In the beginning, Jiangsu Academy of Agricultural Sciences used 'Elberta' and 'Early Crawford' as female parents, and 'Phillips' and 'Tuscan' as the males. Many hybrid seedlings with yellow, clingstone, and flesh texture between hard-melting and nonmelting were obtained, of which the late-maturing 'Jin Feng' and 'Jin Cheng' were released (Wang Zuhua et al., 1989). Shanghai Academy of Agricultural Sciences used 'Bai Hua' and 'Yun Shu 1' (both white flesh) as parents and released the late-maturing 'Yin Xiu', with yellow and melting flesh, for dessert as well as canning (Zhuang Enji et al., 1985). Dalian Agricultural Research Institute carried out selection from open-pollinated seedlings of a local yellow cultivar, Wan Huang Jin, and released the midmaturing 'Feng Huang' and 'Lian Huang'. These two cultivars, with yellow, nonmelting flesh and clingstone, spread widely and became the leading cultivars for canningpeach production in the early 1980s (Wang Zuhua et al., 1989). At the same time, several cultivars for canning were introduced from Japan, such as 'Kanto 5', 'Kanto 14', and 'Myojyo'.

The area under peach cultivation increased rapidly in the late 1970 s, but most leading cultivars matured within a rather short pe- 
riod, as 'Feng Huang', 'Lian Huang', 'Myojyo' and many others are midmaturing. Therefore, the second stage of the breeding programs for canning peach began, with the objective of selecting a complete set of new cultivars with successional maturity (from early to late), in addition to the goals set in the first stage. In this stage (from the late 1970s to present), many excellent $F_{1}$ and $F_{2}$ hybrid seedlings were obtained. Meanwhile, much useful germplasm for canning peaches has been introduced from the United States and Europe, including the 'Babygold' series and NJC selections. With the cooperation of related agencies engaging in peach breeding, two sets of cultivars with successional maturity were released by the mid-1980s for northern or eastern China, respectively, and are being used on a commercial scale in most areas suitable for peach production (Wang Zuhua et al., 1989) (Table 2). In the seventh Five-Year Plan (1986-90), supported by the Ministry of Agriculture, Jiangsu Academy of Agricultural Sciences, Zhengzhou Fruit Research Institute, Zhejiang Academy of Agricultural Sciences, Shanghai Academy of Agricultural Sciences, Dalian Agricultural Research Institute, and Beijing Academy of Agricultural and Forestry Sciences continue to develop yellow peaches for canning. Owing to the wide use of new canning cultivars released through this program, these agencies have evaluated cultivar adaptability in various ecological regions and tested their processing characteristics with the help of canning factories (Hu Zhengling et al., 1990; Wang Zuhua et al., 1990d; Zhang Guirong et al., 1989; Zong Xuepu et al., 1989).
Along with cultivar development, breeders have studied the inheritance of the main traits, such as tree habit, flower type, pollen fertility, fruit shape and weight, flesh color and texture, flavor, stone adhesion, time of bloom and maturity (Shen Dexu, 1980; Wang Zuhua and Duan Xinmei, 1964). Many white peaches, such as 'Bai Hua', are heterogeneous for flesh-color genotype, and new yellow peach cultivars, such as 'Jin Xiu', have been obtained from white-flesh germplasm (Zhuang Enji et al., 1980). Tree habit, flesh color, and flower type were further proved to be each controlled by a pair of alleles and appeared to follow the law of independent assortment, without linkage or interaction among them; further, new dwarf cultivars with the yellow and nonmelting flesh for canning can be selected from $\mathrm{F}_{2}$ hybrids of 'Shouxing Tao' (Prunus persica var. densa Mak.) (Wang Zuhua et al., 1990c).

According to the goals made by the National Peach Breeding Cooperation Organization, Chinese peach breeders will continue to improve peaches. In dessert peach breeding, new programs have already been conducted to develop nectarines, flat peaches and dwarf peaches (Wang Zuhua et al., 1990c; Yu Chengzhe et al., 1990; Zuo Qinyuan et al., 1987). This research will be continued, with the emphases on improving fruit quality and ecological adaptability for nectarines, productivity for flat peaches, and fruit quality for dwarf peaches. In canning-peach breeding, the emphasis will be placed on developing new cultivars with excellent processing characteristics, especially for early maturing ones. Although few attempts have been made in peach breeding for resistance to environmental stress, diseases, and pests (Zuo Qinyuan et al., 1988), their potential importance in production is of concern. Breeders will attempt to initiate multidisciplinary approaches needed to develop peach cultivars or rootstocks with cold hardiness, waterlogging tolerance, and resistance to gummosis and root knot nematodes (Wang Zuhua et al., 1989). Ever greater achievements of peach breeding in China can be anticipated in the future.

\section{Literature Cited (All citations in Chinese)}

Chen Qihui and Gao Min. 1981. Breeding of new peach varieties. China Fruits 1981(1):25-28.

Hu Niyun, Lu Guangming. 1988. Breeding of peach variety 'Xi Nong Zao Mi'. China Fruits 1988(2):10-11.

Hu Zhengling, Wu Shunfa, and Hong Yan. 1981. The techniques for embryo culture of early-maturing peaches. China Fruits 1981(3):35-37.

Hu Zhengling, Wang Jine, Wang Xinfa. 1989. A new early ripening peach variety 'Xue $\mathrm{Yu} \mathrm{Lu'.}$ China Fruits 1989(2):26-27.

Hu Zhengling, Wang Jine, and Wang Xinfa. 1990. A new early ripening yellow peach variety 'Zhe Jin 1'. China Fruits 1990(2):17-18.

Li Zai-Long. 1984. Peach germplasm and breeding in China. HortScience 19(3):348-351.

Li Zengren, Gao Suozhu, and Guo Zhenhuai. 1990. A new early ripening peach variety 'Xin Xing'. China Fruits 1990(4):18-19.

Shen Dexu. 1980. Fruit breeding. Shanghai Sci. and Technol. Publ. House. p. 283-310.

Shen Dexu, Cheng Bingcheng, and Gong Xueli. 1962. Investigation on the variation and selection of Yu Lu peach seedlings. Acta Hort. Sinica 1(2):117-128.

Table 2. Characteristics of new canning peach strains released in China.

\begin{tabular}{|c|c|c|c|c|c|c|c|}
\hline Strains ${ }^{z}$ & Parentage & Maturity & $\begin{array}{l}\text { Mean } \\
\text { fruit wt } \\
\text { (g) }\end{array}$ & Flesh ${ }^{y}$ & $\begin{array}{c}\text { Soluble } \\
\text { solids } \\
\text { concn }(\%)\end{array}$ & Adaptabilityx & Released byy \\
\hline $1-9-5$ & Feng Huang $\times$ Kanto 5 & E & 123 & Y(7).NM.C. & $9-12$ & NC & ZFRI \\
\hline $1-9-12$ & Cheng Yan $\times$ Kanto 5 & $\mathbf{E}$ & 152 & Y(7).NM.C. & $8.5-11.0$ & NC & ZFRI \\
\hline $1-19-4$ & Feng Huang op. & $\mathbf{E}$ & 124 & Y(7).NM.C. & 11.5 & NC & BAAFS \\
\hline $1-7-13$ & Kanto $5 \times$ Feng Huang & $\mathbf{M}$ & 203 & Y(7).NM.C. & $10.0-11.4$ & NC & ZFRI \\
\hline $1-7-9$ & Kanto 5 x Lian Huang & $\mathbf{M}$ & 120 & Y(7).NM.C. & $8-10$ & $\mathrm{NC}$ & ZFRI \\
\hline $3-20-5$ & Kanto $14 \times$ Lian Huang & $\mathbf{M}$ & 148 & Y(9).NM.C. & 10.7 & NC & BAAFS \\
\hline $2-8-13$ & Feng Huang $\times$ Kanto 14 & $\mathbf{M}$ & 170 & Y(8).NM.C. & 13.0 & NC & BAAFS \\
\hline $12-28$ & Shen Zhou Mi Tao x Elberta & $\mathbf{M}$ & 180 & Y(8).NM.C. & 9.8 & NC & DARI \\
\hline $22-6$ & Huang Jin Tao $\times$ Phillips & L & 179 & Y(8).NM.C. & $9.7-10.6$ & NC & DARI \\
\hline $1-6-23$ & Kanto 14 x Lian Huang & $\mathbf{L}$ & 138 & Y(8).NM.C. & 12.8 & $\mathrm{NC}$ & ZFRI \\
\hline $1-7-21$ & Kanto 5 x Lian Huang & L & 196 & Y(8).NM.C. & 14.0 & NC & ZFRI \\
\hline $6-52$ & Xiang Jiao Tao op. & $\mathbf{L}$ & 190 & Y(8).NM.C. & 10.2 & NC & DARI \\
\hline $22-8$ & Huang Jin Tao $\times$ Phillips & $\mathbf{L}$ & 192 & Y(8).NM.C. & $8.8-11.7$ & NC & DARI \\
\hline $6-2-22$ & Kanto $5 \times$ Lian Huang & $\mathbf{E}$ & 105 & Y(7).NM.C. & $8-9$ & SC & ZAAS \\
\hline $5-8-23$ & Feng Huang $\times$ Kanto 14 & $\mathbf{E}$ & 117 & Y(8).NM.C. & $7-8$ & SC & ZAAS \\
\hline $6-1-29$ & Kanto $5 \times$ Feng Huang & $\mathbf{M}$ & 130 & Y(8).NM.C. & $9-10$ & SC & ZAAS \\
\hline $5-24-18$ & Feng Huang $\times$ Tuscan & $\mathbf{M}$ & 120 & Y(8).NM.C. & 10 & $\mathrm{SC}$ & ZAAS \\
\hline $77-13-12$ & Kanto $5 \times$ Feng Huang & M & 134 & Y(7).NM.C. & $9.0-10.2$ & SC & JAAS \\
\hline $77-13-16$ & Kanto $5 \times$ Feng Huang & $\mathbf{M}$ & 148 & Y(8).NM.C. & $11.2-12.6$ & SC & JAAS \\
\hline $6-5-19$ & Kanto $14 \times$ Lian Huang & L & 143 & Y(7).NM.C. & $7-9$ & SC & ZAAS \\
\hline $77-20-5$ & Jin Feng $\times$ Myojyo & L & 138 & Y(8).NM.C. & $10.8-12.4$ & SC & JAAS \\
\hline $77-26-7$ & Jin Cheng $\times$ Kanto 14 & $\mathbf{L}$ & 130 & Y(9).NM.C. & $9.5-13.0$ & SC & JAAS \\
\hline $77-23-9$ & Qiu Kui x Kanto 5 & $\mathbf{L}$ & 175 & Y(8).NM.C. & $10.2-13.4$ & SC & JAAS \\
\hline $77-17-4$ & Phillips $x$ Kanto 5 & $\mathbf{L}$ & 140 & Y(9).NM.C. & $11.0-14.2$ & $\mathrm{SC}$ & JAAS \\
\hline $77-15-2$ & Kanto $14 \times$ Jin Feng & EL & 205 & Y(8).NM.C. & $11.4-13.4$ & SC & JAAS \\
\hline $77-9-2$ & Myojyo X Jin Feng & $\mathrm{EL}$ & 148 & Y(8).NM.C. & $13.0-15.0$ & SC & JAAS \\
\hline
\end{tabular}

${ }^{2}$ Some strains have been named recently, i.e., 'Zheng Huang 2' for 1-9-5; 'Zheng Huang 4', for 1-7-13; 'Yan Feng' for 2-8-13; 'Gui Huang' for 22-6; 'Ju Huang' for 22-8; 'Zhe Jin 2' for 6-22-22; 'Jin Xu' for 77-13-12; 'Jin Hui' for 77-13-16.

see Table 1.

${ }^{x} \mathrm{NC}$, suitable for northern China; SC, suitable for southern China. 
Wang Zuhua. 1988. 'Zao Hua Lu', a very early ripening variety of peach. China Fruits $1988(2): 1-3$.

Wane Zuhua and Duan Xinmei. 1964. Inheritance of some fruit traits in hybrid seedlings of peach cultivars. Acta Hort. Sinica 3(1):11-15.

Wang Zuhua, Lu Zhenxiang, and Hu Zhengling. 1989. Advances and achievements of scientific research on peach breeding and culture in China. China Fruits 1989(4):1-5.

Wang Zuhua, Lu Zhenxiang, and Zhou Jiantao. 1990a. Peach varieties. Agr. Publ. House. p. 1-279.

Wang Zuhua, Lu Zhenxiang, and Lu Xiuhua. 1990b. Study on the evolution and classification of peach varieties through isozyme analysis. Acta Hort. Sinica 17(4):241-248.

Wang Zuhua, Lu Zhenxiang, and Tang Xiulian. 1990c. Inheritance of some qualitative traits in dwarf peach. Jiangsu J. Agr. Sci. 6(Suppl.):4653.

Wang Zuhua, Tang Xiulian, and Guo Hong. 1990d. Two mid-summer yellow peach varieties for canning 'Jin Xu' and 'Jin Hui'. China Fruits 1990(2):19-21.

Yang Zenhai, Hu Niyun, and Lu Guangming. 1983. Study on the embryo culture techniques of early maturing peach in vitro. Acta Collegii Septen-
trionali-Occidntai Agriculturae 1983(1):15-25.

Yao Qiang, Hu Niyun, and Yang Zenhai. 1988. A preliminary research on in-ovulo embryo culture techniques of early-maturing peach. J. Fruit Sci. 5(4):164-168.

Yu Chengzhe and Zong Xuepu. 1980. A survey of germplasm for canning peach in Northwest China. China Fruits 1980(3):18.

Yu Chengzhe, Han Mingyu, and Tian Yuming. 1990. 'Xin Hong Zao Pan Tao', an excellent early ripening variety of flat peach. China Fruits 1990(1):20-21.

Zhang Guirong, Zong Xuepu, and Shen Yunsheng. 1989. A new early-maturing yellow canning-peach variety 'Zheng Huang 205'. J. Fruit Sci. 6(3):118-120.

Zhu Jijin, 'Wu Heming, and Wang Zuhua. 1983. Embryo culture of early-maturing peach cultivars. Jiangsu Agr. Sci. 9:33-35.

Zhu Jijin, Wang Zuhua, and Cai Xinyuan. 1985. Young embryo culture techniques for super-early maturing peaches. Jiangsu J. Agr. Sci. 1(3):4143.

Zhuang Enji, Wu Yuliang, and Xu Zhuying. 1985. 'Jin Xiu', a new variety of yellow peach. China Fruits 1985(3):29-30.

Zhuang Enji, Wu Yuliang, and Xu Zhuying. 1987. 'Chun Lei', a very early ripening variety of peach and its performance in China. China Fruits 1987(1):4-7.

Zhuang Enji, Wu Yuliang, and Cai Xiping. 1980. Studies on the heredity of several characters of White Flower peach. Acta Hort. Sinica 7(2):15.

Zhuang Enji, Xu Zhuying, and Wu Yuling. 1982. The breeding of very early-ripening peaches. Acta Hort. Sinica 9(3):1-6.

Zhuang Enji and Yao Qiang. 1990. Studies on inovulo embryo culture techniques of early-maturing and very early-maturing peaches. Proc. VIth Annu. Natl. Meeting of CSHS. p. 99-102.

Zong Xuepu, Zhang Guirong, and Shen Yunsheng. 1989. A new early ripening yellow peach variety for canning 'Zheng Huang 3'. China Fruits 1989(3):17-19.

Zuo Qinyuan, Gong Fangcheng, and Zhu Gengrui. 1988. Preliminary evaluation on resistance of different peach rootstocks to root knot nematodes. J. Fruit Sci. 5(3):116-119.

Zuo Qinyuan, Zong Xuepu, and Zhu Gengrui. 1987. A preliminary report of observation in introducing nectarines. China Fruits 1987(1):3840.

Zuo Xiangquan and Wu Yongli. 1989. A new early ripening peach variety 'Tuan Cheng Zao Sheng'. China Fruits 1989(1):21-22. 